\section{Introduction to the Dossier Issue "Studying human-computer interaction in translation and interpreting: software and applications}

\author{
Maarit Koponen \\ Lucas Nunes Vieira \\ Nicoletta Spinolo
}

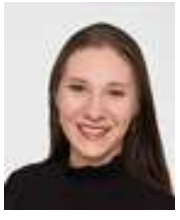

Koponen, Maarit

University of Eastern Finland maarit.koponen@uef.fi; ORCID:

0000-0002-6123-5386

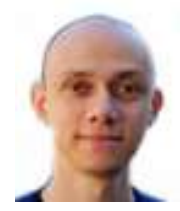

Nunes Vieira, Lucas University of Bristol l.nunesvieira@bristol.ac.uk; ORCID: 0000-0003-3038-4001

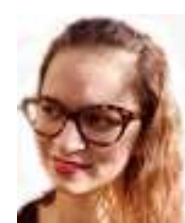

Spinolo, Nicoletta University of Bologna nicoletta.spinolo@unibo.it; ORCID:

0000-0002-5515-13890 interpreting studies. traducció i interpretació.

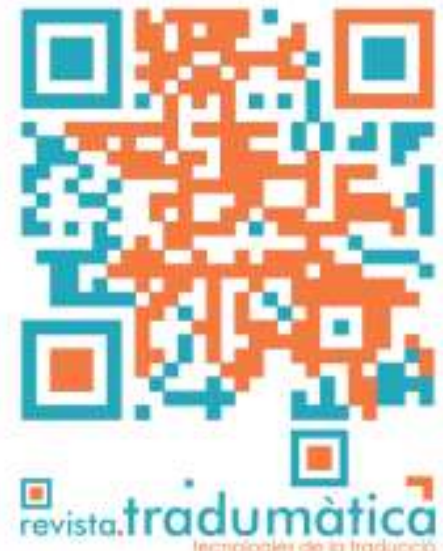

Abstract

Digital tools are changing not only the process of translating and interpreting, but also the industry as a whole, societal perception of and research in translation and interpreting. This Tradumàtica Special Issue collects research on some of these topics, highlighting the importance of furthering research on human-computer interaction in translation and

Keywords: human-computer interaction, natural language processing, translation and interpreting studies, machine translation, spoken data, translation technology, speech technology

Resum

Les eines digitals no només estan canviant la manera de traduir i interpretar, sinó també la indústria en el seu conjunt, la percepció de la societat i la recerca en traducció i interpretació. Aquest número especial de Tradumàtica recull investigacions sobre alguns d'aquests temes, destacant la importància d'aprofundir en la investigació sobre la interacció persona-ordinador en els estudis de

Paraules clau: interacció persona-ordinador, processament del llenguatge natural, estudis de traducció i interpretació, traducció automàtica, dades de veu, tecnologies de la traducció, tecnologies de la parla

\section{Resumen}

Las herramientas digitales no solo están cambiando la manera de traducir e interpretar, sino también la industria en su conjunto, la percepción de la sociedad y la investigación en traducción e interpretación. Este número especial de Tradumàtica recoge investigaciones sobre algunos de estos temas, destacando la importancia de profundizar en la investigación sobre la interacción personaordenador en los estudios de traducción e interpretación.

Palabras clave: interacción persona-ordenador, procesamiento del lenguaje natural, estudios de traducción e interpretación, traducción automática, datos de voz, tecnologías de la traducción, tecnologías del habla 
Advances in natural language processing and related technologies have increasingly important implications for translation, interpreting and cross-cultural communication. Digital tools can change not only the translation and interpreting processes, but also how tasks are managed and commissioned in the language industry, how society interacts with and perceives language technologies, as well as how translation and interpreting research is conducted. This dossier issue of Tradumàtica provides a snapshot of research on some of these topics. The issue set out to examine a range of pressing themes in translation, interpreting and human-computer interaction including use patterns and implications of integrated technologies and multimodal computer-assisted translation, the effects of technological tools and platforms on collaborative translation workflows, and new tools or methodologies for analysing human translation and interpreting. The issue's individual contributions touch on these and several other themes that from the outset were central to the objective of the dossier. The articles also bear witness to important recent events that have the power to shape research priorities and change research practices. The Covid-19 pandemic has unsurprisingly posed obstacles to human-centred research on language technologies, but it has also given strength to new topics and methodologies. Remote settings and technology-mediated interactions are likely here to stay, with growing importance and potentially far-reaching effects on the language industry as well as on research in this area (see Conde Ruano and Artegiani in this issue).

The language industry as a whole has seen considerable changes due to digitalisation and technologisation. Translation workflows became "increasingly technology-embedded" in the 2000s (Doherty 2016: 953), and the rapid rise of neural machine translation in the past few years as well as other technological developments have led to further changes. Implementation and effects of technology have, however, varied across areas of specialisation. In fields such as technical translation and localisation, technology has played a large role already for decades, whereas translation technology has so far had less impact in other areas such as literary translation (Taivalkoski-Shilov 2019; see also Salmi and şahin \& Gürses in this issue).

Technological developments have of course brought benefits. Many find various tools and resources useful in translation work, and cloud-based platforms, for example, can offer opportunities for finding jobs, for collegial discussion and for information sharing (García 2015: 24). On the other hand, technology-mediated workflows can also be seen as an example of "digital Taylorism" (Moorkens 2020), where translation projects are sliced into small pieces and translators' performance is constantly measured. Such fragmentation coupled with automation of various aspects of translation project management, from job allocation to quality assessment, can have a range of adverse effects including downward pressure on remuneration (see e.g. García 2015, 2017) as well as broken communication and ineffective management of projects (see Sakamoto 2019; Artegiani in this issue). Problems can also arise from mismatched technologyrelated expectations between translators, managers and clients (Vieira \& Alonso 2020). 
Implementing technology and human-computer interaction in workflows is not just a technical but a socio-technical process (see Cadwell et al. 2018), which requires further research to provide a more detailed and nuanced picture of diverse translation and interpreting perspectives.

In relation to post-editing of machine translation specifically, we highlight two key aspects of how research is evolving. First, although this is a growing sub-field of research with an already strong body of work, the post-editing literature still has important gaps. Differences in post-editor behaviour, for example, have been observed in many studies (like Alvarez-Vidal, Oliver González \& Badia in this issue), but large-scale, systematic research of what prompts these behaviours and what they mean for translation cognition more broadly is still lacking. Second, uses of machine translation can take different shapes, so the process of editing the machine translation output can unsurprisingly veer in different directions. As machine translation becomes even more widely available across languages, cultures and devices, the concept of machine translation literacy (Bowker \& Buitrago Ciro 2019) is increasingly central to using machine translation safely and effectively. Awareness of machine translation's limitations, and the ability to identify its specific mistakes, is important for language students (Bowker 2020; see also Loock \& Léchauguette in this issue) as well as non-translation professionals (see Vieira, O'Hagan, \& O'Sullivan 2020; Scott \& O'Shea 2021; Asscher \& Glikson 2021). The implications of post-editing processes in these different contexts are an area of priority for future research.

Technology and human-computer interaction can also support and mediate multimodal communication, particularly in relation to audiovisual content (Martìn-Mor \& Sánchez Gijón 2016; Vercauteren, Reviers \& Steyaert in this issue) and to content related to culture and heritage (museums, buildings, exhibitions; see Jiménez Hurtado et al., 2012; Conde Ruano in this issue). Resources such as machine translation or automatic speech recognition can be applied to this type of content not only to save time and costs for businesses and corporations, but also to expand access to information in a more equitable and fair manner (Nurminen \& Koponen 2020). The increasing variety and sophistication of the devices on which different kinds of content can be accessed offer growing opportunities for accessibility while also opening up new avenues for training and research on the implications of multimodality in terms of reception, human-computer interaction, quality assurance and ethics, among others.

As far as interpreting studies are concerned, the increasing integration of technology into the interpreting workflow has sparked interest among researchers and trainers alike. Interpreting is increasingly likely to involve remote communication technologies, automatic speech recognition and computer-assisted interpreting (CAI) tools (Fantinuoli 2019; Defrancq \& Fantinuoli 2020). Furthermore, technology can also support analyses of interpreted and oral data by scaling up dataset sizes through (partial) automation of data transcription and annotation processes (see Koržinek \& Chmiel in this issue), a promising step towards larger and more representative research samples. Relative to research on the implications of technology for translation, interpreting still appears underrepresented, however. Technologisation and automation are likely to impact the 
interpreting profession in ways that are difficult to predict, and this is an area sorely in need of further research (Fantinuoli 2019; Mellinger 2019).

\section{Articles in this Tradumàtica Dossier Issue}

Contributions to the issue have examined a range of specific subjects and data sources, including translators' perceptions of the role of technology in the language industry, post-editing practices, machine translation literacy and error identification, audio description, and development of multimodal resources and spoken corpus methodologies.

Artegiani examines how technology has affected the provision of services and collaborative work particularly in the case of cloud platforms used for subtitling projects. Based on interviews of six professional subtitlers working as freelancers on various cloud platforms, the article focuses on the respondents' experiences of job assignment and quality management in these technology-mediated environments. Artegiani then discusses how their experiences reflect the effect of technology on information and communication patterns. The findings suggest that the features of the cloud platform discourage or even impede negotiation and interaction between different actors - project managers, subtitlers and quality controllers - undermining trust and collaboration. Artegiani's conclusions highlight the importance of research on organisational and social aspects of the technological environment in order to build sustainable production network.

Vela Valido examines the application of technology, particularly machine learning, to the evaluation and assurance of translation quality. The article covers a selection of tools for a varied range of quality management activities from detection of potential errors in human translations to automatic evaluation and estimation of machine translation quality, from tracking process-related performance indicators in translation service provision to predicting quality-related risks. Vela Valido discusses the potential and future prospects of automatic metrics and other machine learning applications particularly in situations that require fast evaluations of large amounts of data, but also notes that specific aspects of quality, particularly meaning, tone and style, have proven difficult to automate. As Vela Valido cautions, automation may also have adverse impacts on the field, and more research is needed on the implications of technology for translation quality management.

Salmi analyses results of a survey distributed to 450 professional Finnish translators. She also compares these results to those obtained with a similar survey distributed to 165 translation students. In all cases, survey respondents had to rate the extent to which they agreed and disagreed with a series of statements about the roles played by language and translation technologies. She found that in most cases language and translation technologies were regarded primarily as concrete tools of translation production as opposed to, for example, tools of communication. Factor analysis was used to identify five underlying factors in the professional translators' responses. The factors were efficiency, sociability, working culture, communication and learning. These factors were found to divide translators based on their areas of specialisation and other aspects of their individual profiles. For example, business translators tended to give 
higher ratings to 'efficiency' statements whereas younger translators tended to give higher ratings to statements associated with the 'communication' factor. Salmi concludes the paper by suggesting further iterations of the methodology as a way of monitoring the impact of technologies on translators' jobs and working processes.

Alvarez-Vidal, Oliver González and Badia examine human-computer interaction and user behaviour in the MT post-editing process. The article describes a pilot experiment with four post-editors working on statistical and neural MT outputs in the medical domain. Based on an analysis of the corrections made by the post-editors in their study, AlvarezVidal, Oliver González and Badia observe differences in the error patterns of SMT and NMT, which may have implications to how post-editors interact with these different technologies. Furthermore, their analysis of this pilot data points to variability in the post-editing behaviour of the four participants. As Vidal Oliver González and Badia note, more detailed research would be needed on such individual differences in behavioural patterns, which may have implications for post-editing and human-computer interaction more broadly.

Almeida Costa, Castro Ferreira, Pagano and Meira propose a Human-machine cooperation protocol for annotating edits in machine translation output. This is a method to classify edit operations on machine-translated texts with the aim of studying the impact of edit types on the quality of post-edited texts. Their approach combines postediting and quality evaluation by humans with an automated analysis that identifies the edits performed and categorises them into different edit types (word order, punctuation, addition, deletion, morphology, or lexical choice). Based on an experiment where 37 participants post-edited and evaluated machine-translated sentences, Almeida Costa, Castro Ferreira, Pagano and Meira analyse relationships between quality scores and specific types of edits. While they note that some edit types, such as changes to word order, may require more fine-grained human analysis to identify the causes and effects of the change, they find that automation can provide helpful support for the human evaluator in identifying and categorising edits and errors.

Şahin and Gürses conducted a literary English-Turkish MT post-editing task involving eleven translation students and eight professional literary translators. In addition to postediting a text, an excerpt of Great Expectations by Charles Dickens, all participants completed a survey and two of the professional translators were interviewed. Participants could choose their preferred MT systems for the task. Most of them used Google Translate, and all of them were critical of the MT outputs used. The authors noted that MT errors often remained in the post-edited texts or were otherwise a factor in low target-text quality, which was particularly noticeable for student participants. Based on the target texts, survey results and the interviews, şahin and Gürses conclude that participants did not believe MT had a contribution to make to English-Turkish literary translation. The authors argue that MT use led to confusion about the role of the translator in the literary task - editors or translators? - which is likely to have influenced participants' decision to leave the machine translation untouched in passages where it required an intervention. They also argue that MT has not yet changed the meaning of 
literary translation for their participants, who considered literary translation tasks to be predominantly, if not entirely, human driven.

Loock and Léchauguette examine undergraduate students' ability to identify and correct errors in machine translations, an important component of MT literacy. The students were in the third year of an Applied Languages degree in France. A sample of 169 of them participated in an error identification task and a further 89 subsequently completed an anonymous survey. The task was based on French machine translations given to students together with the English source text. The survey asked them details of how they used MT in their studies. Of twenty MT errors preliminarily identified by the instructors, 12 on average remained unchanged by the students. Of errors that were identified, approximately a third were wrongly corrected. The authors also found that accuracy errors were identified more often than fluency errors. In contrast to the task results, $93 \%$ of the those who completed the survey believed they were able to identify errors in machine translations. Loock and Léchauguette call attention to how the limitations of MT should be highlighted to students, who in this case missed MT errors in their own native language. The paper makes some proposals for how MT can be used in translation classes, for example in activities where students are asked to compare different translations of the same source texts.

Vercauteren, Reviers and Steyaert present the results of two pilot studies carried out on the English-Dutch language pair aimed at evaluating the effectiveness of machine translation of audio description, with the main goal of identifying typical errors in machine translation of audio descriptions on three Dutch films. By manually evaluating these machine translations, they aimed at identifying typical errors that seemed to occur the in the language combination studied and, more specifically, identifying to which extent they may depend on the specific features of audio description as a text type. As a matter of fact, they find audio description to be a challenging text type for machine translation, because it is characterised by norms that can vary from one country to another, specific linguistic constructions, a high degree of multimodality and because interpretation of the content heavily relies on context. Vercauteren, Reviers and Steyaert identify potential avenues for further research in machine-translated audio description which, as the authors point out, is deemed to grow as legislation in an increasing number countries is requiring more and more audiovisual content to be made accessible.

Conde Ruano reports on the evaluation of a multilingual and accessible audio guide of the Facultad de Letras of the University of the Basque Country, aimed at supporting people with visual impairments in getting to know and moving around the building. Besides describing its creation through a service-learning project involving a collaboration of teachers and students, the paper focuses mainly on the evaluation of the audio guide both in terms of the elaboration process and of the product, suggesting a protocol for the assessment of this product type in terms of compliance with recommendations and standards in the field and of functionality, through a testing and evaluation session that placed special focus on the target users' interaction with the online platform containing the guide and on the learning process involved in the whole project, from product creation to testing and evaluation. 
Koržinek and Chmiel identify a typical challenge in the annotation of interpreting and spoken corpora in general: the identification of different speakers in the dataset. The Polish Interpreting Corpus (PINC) is an interpreting corpus of European Parliament Polish or English original speeches and their respective interpretations, and it includes over 190,000 tokens. Koržinek and Chmiel present a method employed on PINC for automatically identifying voices using a deep neural network model, and offer a complete step-by-step tutorial for implementing the protocol, thus offering scholars working on interpreting and on spoken corpora in general new insight into ways to perform speaker identification in an automatic and effective way. This implementation of technology speeds up an annotation process that is known to be complex and time-consuming and paves the way for faster annotation of increasingly large corpora.

The contributions to this Tradumàtica dossier issue highlight the growing diversity of technology-mediated practices in the language industry and the importance of further study of human-computer interaction for translation and interpreting. We hope this issue will stimulate discussion and new directions for research in this area.

\section{References}

Asscher, Omri; Glikson, Ella. 2021. "Human evaluations of machine translation in an ethically charged situation." New Media \& Society. 〈https://journals.sagepub.com/doi/abs/10.1177/14614448211018833〉 https://doi.org/10.1177/14614448211018833 [Accessed: 20211222]

Bowker, Lynne; Buitrago Ciro, Jairo. 2019. Machine Translation and Global Research: Towards Improved Machine Translation Literacy in the Scholarly Community. Bingley: Emerald Publishing Limited. 〈https://doi.org/10.1108/9781787567214〉 [Accessed: 20211222]

Bowker, Lynne. 2020. "Machine translation literacy instruction for international business students and business English instructors." Journal of Business \& Finance Librarianship 25 (1-2): 25-43. 〈https://doi.org/10.1080/08963568.2020.1794739〉. [Accessed: 20211222]

Cadwell, Patrick; O’Brien, Sharon; Teixeira, Carlos S.C. 2018. "Resistance and Accommodation: Factors for the (Non-) Adoption of Machine Translation among Professional Translators." Perspectives: Studies in Translatology 26 (3): 301-21. 〈https://doi.org/10.1080/0907676X.2017.1337210〉. [Accessed: 20211222]

Defrancq, Bart; Fantinuoli, Claudio. 2020. "Automatic speech recognition in the booth: Assessment of system performance, interpreters' performances and interactions in the context of numbers". Target 33:1, 73-102. 〈https://doi.org/10.1075/target.19166.def〉. [Accessed: 20211222]

Doherty, Stephen. 2016. "The Impact of Translation Technologies on the Process and Product of Translation.” International Journal of Communication 10: 947-69. 
Fantinuoli, Claudio. 2019. "The technological turn in interpreting: the challenges that lie ahead". Proceedings of the conference Übersetzen und Dolmetschen 4.0. - Neue Wege im digitalen Zeitalter, 334-354.

García, Ignacio. 2015. "Cloud Marketplaces: Procurement of Translators in the Age of Social Media." Journal of Specialised Translation, no. 23: 18-38.

García, Ignacio. 2017. "Translating in the Cloud Age: Online Marketplaces." Hermes: Journal of Language and Communication Studies, no. 56: 59-70. 〈https://doi.org/10.7146/hjlcb.v0i56.97202〉. [Accessed: 20211222]

Jiménez Hurtado, Catalina; Seibel, Claudia; Soler Gallego, Silvia. 2012. "Museos para todos: la traducción e interpretación para entornos multimodales como herramienta de accesibilidad universal". MonTI. Monografías de Traducción e Interpretación, (4), 349-383. 〈https://doi.org/10.6035/MonTI.2012.4.15〉. [Accessed: 20211222]

Martìn-Mor, Adrià; Sánchez-Gijón, Pilar. 2016. "Machine translation and audiovisual products: a case study". JoSTrans, (26), 172-186. 〈https://jostrans.org/issue26/art_martin.php〉. [Accessed: 20211222]

Mellinger, Christopher D. 2019. "Computer-assisted interpreting technologies: a product and process-oriented perspective". Revista Tradumàtica 17:33-44. 〈https://doi.org/10.5565/rev/tradumatica.228〉. [Accessed: 20211222]

Moorkens, Joss. 2020. “'A Tiny Cog in a Large Machine': Digital Taylorism in the Translation Industry." Translation Spaces 9 (1): 12-34. 〈https://doi.org/10.1075/ts.00019.moo〉. [Accessed: 20211222]

Nurminen, Mary; Koponen, Maarit. 2020. "Machine translation and fair access to information". Translation Spaces 9:1, 150-169. < https://doi.org/10.1075/ts.00025.nur>. [Accessed: 20211222]

Sakamoto, Akiko. 2019. "Unintended Consequences of Translation Technologies: From Project Managers' Perspectives.” Perspectives 27 (1): 58-73. 〈https://doi.org/10.1080/0907676X.2018.1473452〉. [Accessed: 20211222]

Scott, Juliette; O’Shea, John. 2021. "How Legal Documents Translated Outside Institutions Affect Lives, Businesses and the Economy." International Journal for the Semiotics of Law-Revue internationale de Sémiotique juridique: 1-43. 〈https://doi.org/10.1007/s11196-020-09815-5〉. [Accessed: 20211222]

Taivalkoski-Shilov, Kristiina. 2019. "Ethical Issues Regarding Machine(-Assisted) Translation of Literary Texts." Perspectives: Studies in Translation Theory and Practice 27 (5): 689-703. 〈https://doi.org/10.1080/0907676X.2018.1520907〉. [Accessed: 20211222]

Vieira, Lucas Nunes, O’Hagan, Minako; O'Sullivan, Carol. 2020. "Understanding the societal impacts of machine translation: a critical review of the literature on medical and legal use cases." Information, Communication \&amp; Society: 1-18. 〈https://doi.org/10.1080/1369118X.2020.1776370〉. [Accessed: 20211222] 
Vieira, Lucas Nunes; Alonso, Elisa. 2020. "Translating Perceptions and Managing Expectations: An Analysis of Management and Production Perspectives on Machine Translation." Perspectives: Studies in Translation Theory and Practice 28 (2): 163-84. 〈https://doi.org/10.1080/0907676X.2019.1646776〉.[Accessed: 20211222] 